\section{MR Imaging: An Increasingly Important Tool in the Early Diagnosis of Wernicke Encephalopathy}

The recent article by Ha et al published in the American Journal of Neuroradiology ${ }^{1}$ has rekindled the debate sparked by previous studies $^{2-4}$ that had linked atypical brain MR imaging findings to the nonalcoholic (NA) variant of Wernicke encephalopathy (WE). In unselected patients with WE, the anatomic regions most frequently involved by MR imaging are the medial thalami and the periventricular regions of the third ventricle. ${ }^{3}$ However, atypical MR imaging findings may also be observed, including symmetric alterations of the cerebellum, cerebral cortex, and cranial nerve nuclei $(\mathrm{CNN}) .{ }^{3,4}$ Atypical MR imaging findings usually occur in association with the typical MR imaging findings of WE. ${ }^{5}$ In the largest case series published to date encompassing 56 patients with WE, we detected atypical MR imaging changes more commonly in NA compared with patients with alcoholism (AL). ${ }^{4}$ In particular, involvement of $\mathrm{CNN}$ was seen in $32 \%$ of NA versus none of the AL patients.

In contrast to our findings, $\mathrm{Ha}$ et $\mathrm{al}^{1}$ found no significant differences in the distribution of typical and atypical MR imaging findings in AL and NA patients with WE. Both their ${ }^{1}$ and our studies are retrospective in design, and thus prone to incurring a selection bias due to the lack of predefined entry criteria. Therefore, the study populations may differ in some characteristics that cannot be determined a posteriori.

There are, however, some differences in the studies that may help to explain, at least in part, their different conclusions. First, our study included more than twice as many patients as the study by Ha et al. ${ }^{1}$ Sample size is invariably a critical issue because all other things being equal, studies with larger numbers of patients are more likely to arrive at significant results than those with fewer patients. In particular, if we consider only NA patients, our study included 3 times as many patients $(n=30)$ as the study by Ha et al $(n=11)$. Second, in our patients, MR imaging was performed in the acute stage of WE, whereas in the study by $\mathrm{Ha}$ et al, the mean interval between the onset of manifestations and performance of MR imaging was 4.5 days. This delay may have potentially resulted in some differences in the brain changes depicted by MR imaging. Third, $43 \%$ of our patients fulfilled the classic diagnostic triad of WE compared with $17 \%$ of the patients investigated by $\mathrm{Ha}$ et al, suggesting that our patients may have had, on average, more severe disease and thus a higher likelihood of having abnormal MR imaging findings. Fourth, Ha et al did not specifically identify CNN lesions except in 1 NA patient with involvement of CNN IX. However, they did show that $14 \mathrm{NA}$ patients and $10 \mathrm{AL}$ patients had active lesions in the dorsal medulla or in the periventricular gray matter of the fourth ventricle, which are sites where many CNN are located. ${ }^{1}$ Therefore, although CNN were not formally identified in the study by $\mathrm{Ha}$ et $\mathrm{al}^{1}{ }^{1}$ the findings of their study may, nevertheless, support the concept that CNN involvement is more common in NA patients, in agreement with our own ${ }^{3,4,6}$ and the findings of others. ${ }^{2,7}$ The involvement of CNN IX reported by Han et al is a particularly intriguing finding, both because this specific CNN has not previously been shown to be involved in $\mathrm{WE}^{4,8}$ and because CNN IX is notoriously difficult to distinguish from neighboring nuclei. It would be interesting to have a chance to review the images on the basis of which CNN IX involvement was diagnosed.

We concur with $\mathrm{Ha}$ et $\mathrm{al}^{1}$ that thiamine deficiency is a crucial component in the pathogenesis of WE and that prompt treatment with thiamine can avert a dismal prognosis. At risk patients should thus receive thiamine regardless of whether they are hospitalized. However, we would also like to suggest that other factors, including alcohol intake, may modulate the expression of WE. ${ }^{5}$ Until data from prospective studies become available, we think that the current body of evidence still supports the notion that MR imaging findings in NA patients differ, at least in part, from those of AL patients with WE. Most important, both our study ${ }^{4}$ and the one by Ha et $\mathrm{al}^{1}$ clearly demonstrate that the spectrum of radiographic alterations is broader than that of "classic" changes. Therefore, radiologists should be aware that WE may present with both typical and atypical findings. At least equally important, despite the partial discrepancies in the results published, the role of MR imaging in clinching an early diagnosis of WE is fully confirmed.

\section{References}

1. Ha ND, Weon YC, Jang JC, et al. Spectrum of MR imaging findings in Wernicke encephalopathy: are atypical areas of involvement only present in nonalcoholic patients? AJNR Am J Neuroradiol 2012 Mar 1. [Epub ahead of print]

2. Bae SJ, Lee HK, Lee JH, et al. Wernicke's encephalopathy: atypical manifestation at MR imaging. AJNR Am J Neuroradiol 2001;22:1480-82

3. Zuccoli G, Gallucci M, Capellades J, et al. Wernicke encephalopathy: MR findings at clinical presentation in 26 alcoholic and nonalcoholic patients. AJNR Am J Neuroradiol 2007;28:1328-31

4. Zuccoli G, Santa CD, Bertolini M, et al. MR imaging findings in 56 patients with Wernicke encephalopathy: nonalcoholics may differ from alcoholics. AJNR Am J Neuroradiol 2009;30:171-76

5. Zuccoli G, Pipitone N. Neuroimaging findings in acute Wernicke's encephalopathy: review of the literature. AJR Am $J$ Roentgenol 2009;192:501-08

6. Zuccoli G, Motti L. Atypical Wernicke's encephalopathy showing lesions in the cranial nerve nuclei and cerebellum. J Neuroimaging 2008;18:194-97

7. Hygino da Cruz LC Jr, Domingues RC, Vilanova I, et al. MR imaging findings in Wernicke encephalopathy: nonalcoholics may be similar to alcoholics. AJNR Am J Neuroradiol 2010;31:E54-55

8. Santos AC, Tavares LL, da Graca Morais MM, et al. Non-alcoholic Wernicke's encephalopathy: broadening the clinicoradiological spectrum. $\mathrm{Br} J$ Radiol 2010;83:437-46

G. Zuccoli

Department of Radiology University of Pittsburgh Medical Center Children's Hospital of Pittsburgh Pittsburgh, Pennsylvania N. Pipitone Rheumatology Unit, Department of Internal Medicine Azienda Ospedaliera ASMN Istituto di Ricovero e Cura a Carattere Scientifico Reggio Emilia, Italy

http://dx.doi.org/10.3174/ajnr.A3152 